\title{
Chronic Pericardial Hematoma with Suppression of the Right Ventricle: A Rare Complication of a Coronary Artery Aneurysm
}

\author{
Markus Schlömicher ${ }^{1}$ Peter Lukas Haldenwang ${ }^{1} \quad$ Josef Reichert $^{1} \quad$ Vadim Moustafine $^{1}$ \\ Matthias Bechtel ${ }^{1}$ Justus Thomas Strauch ${ }^{1}$
}

${ }^{1}$ Department of Cardiothoracic Surgery, RUHR University Bochum, Bochum, North Rhine-Westphalia, Germany

Thorac Cardiovasc Surg Rep 2014;3:27-30.

\begin{abstract}
Address for correspondence Markus Schlömicher, MD, Department of Cardiothoracic Surgery, Hospital Bergmannsheil Bochum, Bürkle de la Camp Platz 1, Bochum, North Rhine-Westphalia 44789, Germany (e-mail: m.schloemicher@gmail.com).
\end{abstract}

\begin{abstract}
We present a case of a 67-year-old patient referred to our department with a pericardial mass lesion measuring $11 \times 4 \times 7.5 \mathrm{~cm}$ as diagnosed in computed tomography scan. The patient showed a history of progredient dyspnea. Video-assisted thoracoscopy as well as an explorative full sternotomy to resect the mass lesion had been performed at the referring hospital subsequently before admission to our department. Intermittent hemodynamic instability caused the procedure to stop and a transfer to the cardiothoracic surgery department, following which a resternotomy was performed. Inspection of the surgical site and subsequent intraoperative rapid section revealed an old organized and dense pericardial hematoma adherent to the right ventricle. The suspicion of covered coronary artery perforation led to an intraoperative coronary angiography, which revealed a large proximal coronary aneurysm of the right coronary artery and a subtotal stenosis of the

Keywords

- aneurysm

- thoracic surgery circumflex branch. The hematoma could be removed with decompression of the right ventricle under cardiopulmonary bypass conditions. The further postoperative course was uncomplicated with retransfer to the referring hospital on the postoperative day 8 .
\end{abstract}

\section{Introduction}

A prevalence of coronary artery aneurysms can be found ranging from 1.2 to $4.9 \%$ in populations undergoing angiography, and $1.4 \%$ in autopsy studies. ${ }^{1}$

Coronary artery ectasia is defined as a localized dilatation with a diameter of $\geq 1.5$ times the adjacent normal coronary artery. ${ }^{2}$ We present a case of a chronic coronary artery aneurysm leading to a significant organized pericardial hematoma causing massive suppression of the right ventricle.

\section{Case Report}

A 67-year-old male patient was referred to our department by a thoracic surgery unit in the periphery. A pericardial mass lesion measuring $11 \times 4 \times 7.5 \mathrm{~cm}$ was found in the computed tomography (CT) scan leading to significant suppression of the right ventricle ( - Fig. 1 ). The patient showed a medical history of progressive dyspnea and weight loss $(>13 \mathrm{~kg}$ ) in the past 6 weeks with a recurrent right-sided pleural effusion. There was no history of thoracic trauma. Echocardiographically, as well as in the CT scan, there was no suspicion of myocardial involvement. The tentative diagnosis at that time was pericardial cyst. A coronary artery aneurysm could not be identified by the radiologist. Video-assisted thoracoscopy, as well as explorative full sternotomy to resect the mass lesion had been performed at the referring hospital subsequently before the admission to our department. The distinctive adjacency to the right ventricle and intermittent hemodynamic instability required the procedure to stop and a transfer to the cardiothoracic surgery department received

February 16, 2014

accepted after revision

March 24, 2014

published online

September 3, 2014
DOI http://dx.doi.org/

10.1055/s-0034-1386720. ISSN 2194-7635. (c) 2014 Georg Thieme Verlag KG
Stuttgart · New York

License terms

(1) $\Theta \circledast$ 


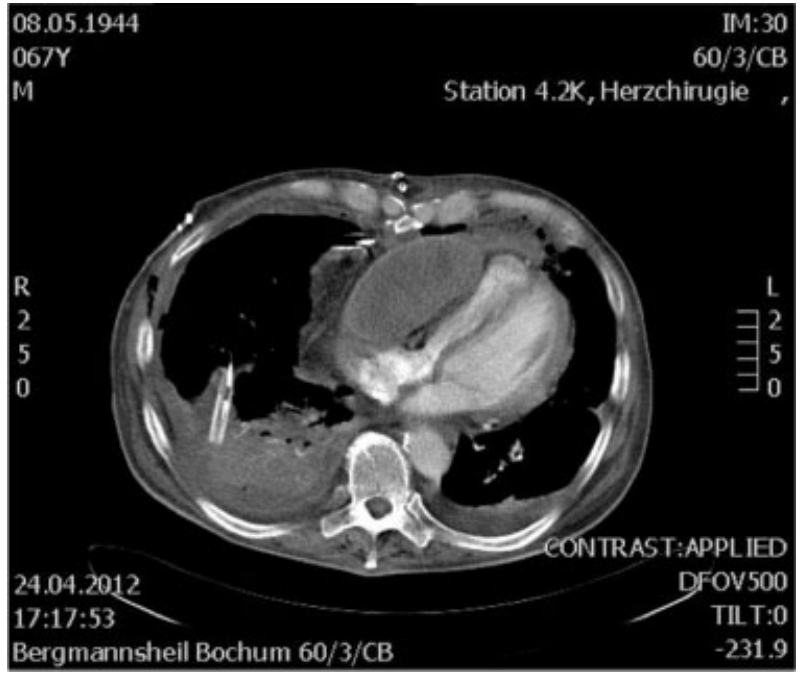

Fig. 1 CT scan showing a pericardial mass lesion with suppression of the right ventricle. CT, computed tomography.

to remove the tumorous mass under cardiopulmonary bypass backup. The intubated and mechanically ventilated patient was transferred in a cardiopulmonary unstable condition with catecholamine support.

An immediate resternotomy was performed. Inspection of the surgical site and subsequent intraoperative rapid section revealed an old organized and dense pericardial hematoma adherent to the right ventricle ( $\mathbf{- F i g}$. 2 ). The operation was performed in a hybrid operating room with the opportunity to perform coronary angiographies. Thus, intraoperative coronary angiography was done, showing a large proximal coronary aneurysm of the right coronary artery (RCA) and a subtotal stenosis of the circumflex branch in the distal third (-Fig. 3). Angiographically there was no evidence of perforation with leakage of the contrast agent. After establishment of cardiopulmonary bypass via direct standard cannulation, using a twostage cannula for venous drainage, the hematoma could be removed with a fully unloaded right ventricle (-Fig. 4).

After the removal, the aneurysm of the RCA was inspected. It showed no bleeding so that a decision against aneurysm resection and bypass grafting was made with regards to an

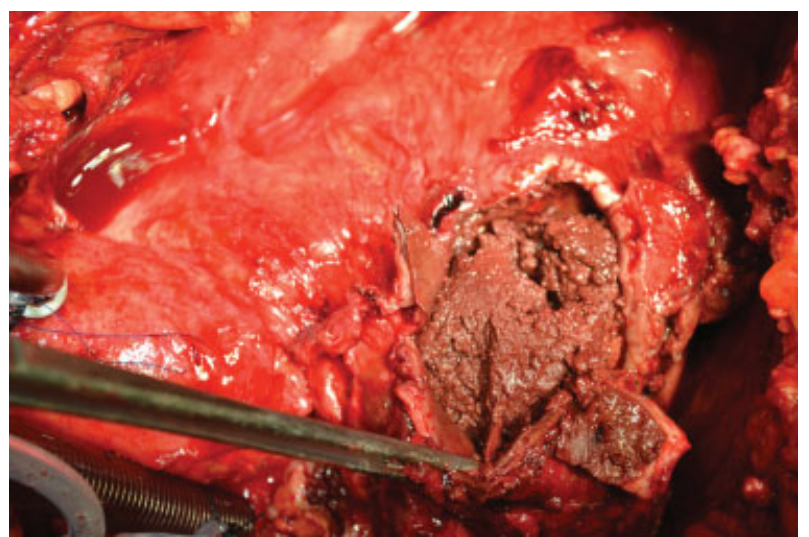

Fig. 2 Operative finding showing a chronic hematoma adherent to the right ventricle.

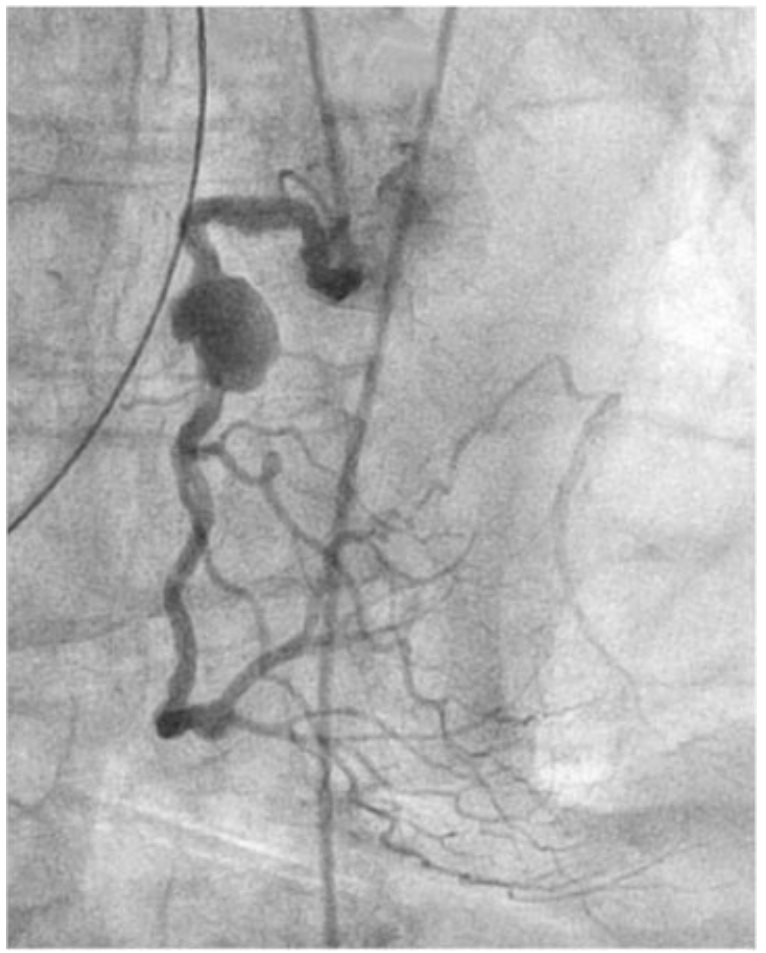

Fig. 3 Intraoperative coronary angiography revealing a large aneurysm of the proximal right coronary artery as well as a subtotal stenosis of the circumflex branch.

overall detectable weakness of the macerated tissue, where the chronic hematoma was adherent ( - Fig. 5). The wall of the right ventricle as well as the aneurysm was sealed with TachoSil (Takeda Pharmaceutical Company Limited, Osaka, Japan). Weaning from cardiopulmonary bypass was unproblematic followed by a transfer to intensive care unit. Further postoperative course was uncomplicated. The patient was retransferred to the referring hospital on postoperative day 8 . Interventional treatment of the RCA aneurysm with a polytetrafluoroethylene (PTFE)-covered stent to exclude the aneurysm is planned after a 3 months period to await consolidation of the tissue. No necessity is seen to treat the ramus circumflexus stenosis in the distal third of the vessel.

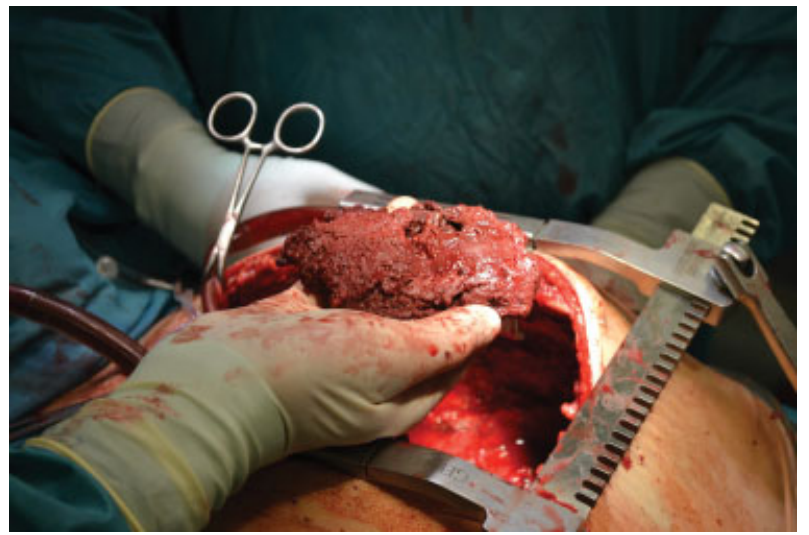

Fig. 4 Intraoperative image of the hematoma after removal. 


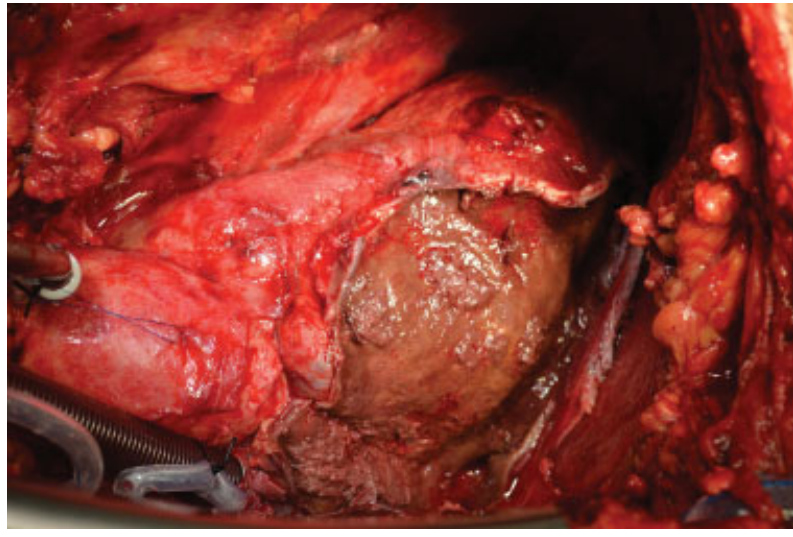

Fig. 5 Intraoperative image of the decompressed right ventricle.

\section{Discussion}

The main sites for coronary artery aneurysms are the proximal and middle segments of the RCA (68\%); the left anterior descending (60\%), and the left circumflex artery (50\%). Coronary artery aneurysms of the main stem are rare. ${ }^{1}$

The most common cause of coronary artery aneurysms is atherosclerosis, but aneurysms may also be congenital, mycotic, or part of a systemic inflammatory disease such as polyarteritis nodosa or the mucocutaneous lymph node syndrome of Kawasaki. ${ }^{1}$ Multiple aneurysms in childhood and adolescence are normally complications of Kawasaki disease. The pathogenesis is rather complex and poorly understood. Extensive inflammation of all vessel wall layers plays a major role in development. ${ }^{3}$ With an increased diameter; increased wall stress causes progressive dilatation of the affected arterial segment. ${ }^{4}$

Patients with coronary artery aneurysms can be asymptomatic but also present with exercise-induced cardiac angina due to myocardial ischemia without coincident significant coronary artery stenosis despite the significant higher volumetric blood flow. This might be linked to a depressed coronary flow reserve, which is considered a result of severe microcirculatory dysfunction. ${ }^{5}$ Additional clinical presentations are myocardial infarction and sudden cardiac death due to complications such as thrombus formation, distal embolization, shunt formation or rupture. ${ }^{6,7}$ Left-to-right shunts to the right atrium, the right ventricle or the coronary sinus, inducing heart murmur and congestive heart failure can occur. ${ }^{4}$

Noninvasive detection of coronary artery aneurysms includes echocardiography, CT, and magnetic resonance imaging. ${ }^{2,8}$ Some rare cases of giant coronary aneurysms can cause a pronounced bulge of the heart counter on chest X-rays. ${ }^{8}$ The gold standard in the quantification of coronary artery aneurysms is represented by invasive coronary angiography, providing information about the size, shape, location, and number of aneurysms. ${ }^{9}$

The differential diagnosis for pericardial mass lesion include graft aneurysms after coronary artery bypass surgery, congenital pericardial cysts, echinococcal cysts, dilated coro- nary sinuses, dilated coronary fistulas, primary cardiac tumors, and metastatic tumors. ${ }^{2}$ Coronary artery aneurysms can be treated conservatively using antiplatelets and anticoagulants, interventionally or surgically.

Interventional strategies include PTFE-covered balloon or self-expandable stents to achieve exclusion of the aneurysm. ${ }^{10,11}$ Surgical therapy should be taken into consideration for large symptomatic aneurysms to avoid further complications such as extension, thrombosis, rupture, or coronary embolization. ${ }^{12}$ Therefore, various surgical strategies have emerged such as reconstruction, resection, or isolation with concomitant bypass surgery. Fistulas should be closed. Compromised coronary blood flow needs bypass surgery to be performed.

It is being discussed if coronary artery aneurysms have an independent adverse effect on long-term survival or if the prognosis is based on the coronary atherosclerosis per se. An overall survival after 5 years is estimated at only $71 \%{ }^{13}$

Organized intrapericardial hematomas are mostly found in association with trauma, cardiac surgery, and coronary interventions. ${ }^{14-16} \mathrm{~A}$ chronic intrapericardial hematoma being a complication of a coronary artery aneurysm has not been described so far which underlines the uniqueness of this case.

This case illustrates the importance of substantial differential diagnostic knowledge of intrapericardial mass lesions before surgery. Surgery on lesions with adherence to cardiac structures, especially the right ventricle, should be performed in cardiothoracic surgery units under cardiopulmonary bypass stand by.

The appropriate therapy of coronary artery aneurysms is uncertain. The discussion regarding surgical versus interventional treatment remains controversial. Some authors even describe conservative treatment options. ${ }^{17}$ We plan the implant a PTFE-covered stent in the near future to avoid cardiac reoperation. A reoperation might be associated with a higher risk in this case especially considering the extensive maceration of the right ventricle wall secondary to the chronic hematoma. With regards to the hematoma being a complication of the coronary artery aneurysm, the interventional exclusion of the aneurysm has to be considered being inevitable to avoid further complications.

\section{References}

1 Nichols L, Lagana S, Parwani A. Coronary artery aneurysm: a review and hypothesis regarding etiology. Arch Pathol Lab Med 2008;132(5):823-828

2 Pahlavan PS, Niroomand F. Coronary artery aneurysm: a review. Clin Cardiol 2006;29(10):439-443

3 Turhan H, Erbay AR, Yasar AS, et al. Plasma soluble adhesion molecules; intercellular adhesion molecule-1, vascular cell adhesion molecule-1 and E-selectin levels in patients with isolated coronary artery ectasia. Coron Artery Dis 2005;16(1):45-50

4 Chia HM, Tan KH, Jackson G. Non-atherosclerotic coronary artery aneurysms: two case reports. Heart 1997;78(6):613-616

5 Akyürek O, Berkalp B, Sayin T, Kumbasar D, Kervancioğlu C, Oral D. Altered coronary flow properties in diffuse coronary artery ectasia. Am Heart J 2003;145(1):66-72 
30 Chronic Pericardial Hematoma with Suppression of the Right Ventricle Schlömicher et al.

6 Anfinsen OG, Aaberge L, Geiran O, Smith HJ, Aakhus S. Coronary artery aneurysms mimicking cardiac tumor. Eur J Echocardiogr 2004;5(4):308-312

7 Dogan A, Ozaydin M, Gedikli O, Altinbas A, Ergene O. Effect of trimetazidine on exercise performance in patients with coronary artery ectasia. Jpn Heart J 2003;44(4):463-470

8 Tanabe M, Onishi K, Hiraoka N, et al. Bilateral giant coronary aneurysms diagnosed non-invasively by dynamic magnetic resonance imaging. Int J Cardiol 2004;94(2-3):341-342

9 Wang KY, Ting CT, St John Sutton M, Chen YT. Coronary artery aneurysms: A 25-patient study. Catheter Cardiovasc Interv 1999; 48(1):31-38

10 Burzotta F, Trani C, Romagnoli E, et al. Percutaneous treatment of a large coronary aneurysm using the self-expandable Symbiot PTFEcovered stent. Chest 2004;126(2):644-645

11 Briguori C, Sarais C, Sivieri G, Takagi T, Di Mario C, Colombo A. Polytetrafluoroethylene-covered stent and coronary artery aneurysms. Catheter Cardiovasc Interv 2002;55(3):326-330
12 Li D, Wu Q, Sun L, et al. Surgical treatment of giant coronary artery aneurysm. J Thorac Cardiovasc Surg 2005;130(3):817-821

13 Baman TS, Cole JH, Devireddy CM, Sperling LS. Risk factors and outcomes in patients with coronary artery aneurysms. Am J Cardiol 2004;93(12):1549-1551

14 Chamsi-Pasha MA, Anwar AM, Nosir YF, et al. Organized intrapericardial haematoma: a rare cause of heart failure after 2 years of trauma. Eur J Echocardiogr 2011;12(1):E1

15 Wechsel M, Ropers D, Ropers U, et al. Organized intrapericardial hematoma after coronary artery bypass surgery. J Cardiovasc Comput Tomogr 2008;2(5):328-331

16 Kawase Y, Hayase M, Ito S, et al. Compression of right ventricular out-flow due to localized hematoma after coronary perforation during PCI. Catheter Cardiovasc Interv 2003;58(2):202-206

17 Khan IA, Dogan OM, Vasavada BC, Sacchi TJ. Nonatherosclerotic aneurysm of the left circumflex coronary artery presenting with accelerated angina pectoris: response to medical management-a case report. Angiology 2000;51(7):595-598 